\title{
Exploring the phase diagram of QCD with complex Langevin simulations
}

\author{
Gert Aarts $^{1}$, Felipe Attanasio ${ }^{1,2}$, Benjamin Jäger ${ }^{* 1}$, Erhard Seiler ${ }^{3}$, Dénes Sexty ${ }^{4,5}$, \\ Ion-Olimpiu Stamatescu ${ }^{4}$. \\ ${ }^{1}$ Department of Physics, College of Science, Swansea University, Swansea, UK \\ ${ }^{2}$ CAPES Foundation, Ministry of Education of Brazil, Brasília, Brazil \\ ${ }^{3}$ Max-Planck-Institut für Physik (Werner-Heisenberg-Institut), München, Germany \\ ${ }^{4}$ Institut für Theoretische Physik, Universität Heidelberg, Heidelberg, Germany \\ ${ }^{5}$ Department of Physics, Bergische Universität Wuppertal, Wuppertal, Germany \\ E-mail: B. Jaeger@swansea.ac.uk,
}

\begin{abstract}
Simulations of QCD with a finite chemical potential typically lead to a severe sign problem, prohibiting any standard Monte Carlo approach. Complex Langevin simulations provide an alternative to sample path integrals with oscillating weight factors and therefore potentially enable the determination of the phase diagram of QCD. Here we present results for QCD in the limit of heavy quarks and show evidence that the phase diagram can be mapped out by direct simulation. We apply adaptive step-size scaling and adaptive gauge cooling to ensure the convergence of these simulations.
\end{abstract}

The 32nd International Symposium on Lattice Field Theory

June 23 - 28, 2014

Columbia University, New York, USA

${ }^{*}$ Speaker. 


\section{Introduction}

The current knowledge of the phase diagram of QCD is very limited and a possible scenario is sketched in figure 1 . The structure of the phase diagram is very important for a wide variety of

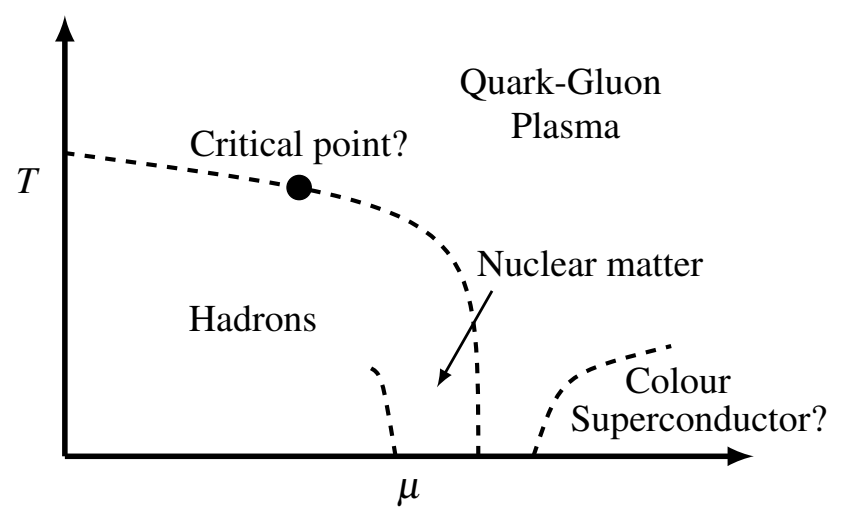

Figure 1: A possible sketch of the QCD phase diagram.

phenomena: the evolution of the early universe, neutron stars and heavy-ion collision experiments. Predictions from first principles are a clearly desirable goal, but unfortunately direct lattice simulations of QCD are not possible due to the famous sign problem [1]: Importance Sampling based Monte Carlo methods cannot be applied to path integrals with a highly oscillating complex weight. For non-vanishing $\mu$, the fermion determinant

$$
[\operatorname{det} D(\mu)]^{*}=\operatorname{det} D\left(-\mu^{*}\right) \rightarrow \operatorname{det} D(\mu \neq 0)=|\operatorname{det} D| \mathrm{e}^{i \Theta} \in \mathbb{C},
$$

adds a complex phase to the path integral,

$$
\langle A\rangle=\frac{1}{Z} \int \mathrm{D} U A(U)|\operatorname{det} D| \mathrm{e}^{i \Theta} \mathrm{e}^{-S_{G}(U)} .
$$

The complex Langevin method has been shown to provide an alternative to perform lattice simulation with a non-vanishing chemical potential [2-12].

\section{Setup}

The complex Langevin method is based on stochastic quantization, in which the degrees of freedom $U$, i.e. the gauge links, are evolved with respect to the so-called Langevin time $t$, suitably discretised,

$$
U(t+\varepsilon)=R(t) U(t) \quad \text { with } \quad R(t)=\exp [i \lambda(-\varepsilon \partial S+\sqrt{\varepsilon} \eta)],
$$

where $\eta$ is Gaussian white noise and $\lambda$ are the Gell-Mann matrices. These gauge fields are complexified by extending the gauge group from $\mathrm{SU}(3)$ to $\mathrm{SL}(3, \mathbb{C})$. With these definitions the expectation value of an observable $A$ can be obtained by integrating along the Langevin evolution

$$
\langle A\rangle=\frac{1}{T} \int_{0}^{T} A[U(t)] \mathrm{d} t .
$$


We apply adaptive step-size scaling [6] and adaptive gauge cooling [7-9] to avoid excursions into the non-compact "imaginary" directions of the larger gauge group $\operatorname{SL}(3, \mathbb{C})$. These excursions are typically generated by numerical artefacts and round-off errors. Convergence of the Langevin method can be proven, if the distribution in the "imaginary" directions shows a strong enough falloff and the action is holomorphic [10,11]. Therefore we monitor the distribution of our results as well as the unitarity norm, which is a measure of the distance of the gauge links from the $\mathrm{SU}(3)$ manifold and given by

$$
\text { unitnorm }=\operatorname{Tr}\left(U U^{\dagger}-\mathbb{I}\right)^{2} \geq 0 .
$$

In the following we present results for QCD in the limit of heavy quarks using the Wilson formulation. The quarks are considered static in this approximation, so that the spatial hopping part is neglected. This approach is known as heavy dense QCD or HDQCD. In this limit the fermion determinant simplifies and can be written in terms of the (conjugate) Polyakov loops $\mathscr{P}_{\vec{x}}$ and $\mathscr{P}_{\vec{x}}^{-1}$ as

$$
\operatorname{det} D(\mu)=\prod_{\vec{x}} \operatorname{det}\left(1+h \mathrm{e}^{\mu / T} \mathscr{P}_{\vec{x}}\right)^{2} \operatorname{det}\left(1+h \mathrm{e}^{-\mu / T} \mathscr{P}_{\vec{x}}^{-1}\right)^{2},
$$

where $h=(2 \kappa)^{N_{\tau}}$. For the gluonic part we use the full Wilson gauge action. Figure 2 shows the real part of the Polyakov loop $P_{\vec{x}}=\operatorname{Tr} \mathscr{P}_{\vec{x}} / 3$ and the unitarity norm as a function of the Langevin time $t$ for a particular simulation $\left(8^{3} \cdot 12, \mu=2.39, \beta=5.8, \kappa=0.04\right)$.
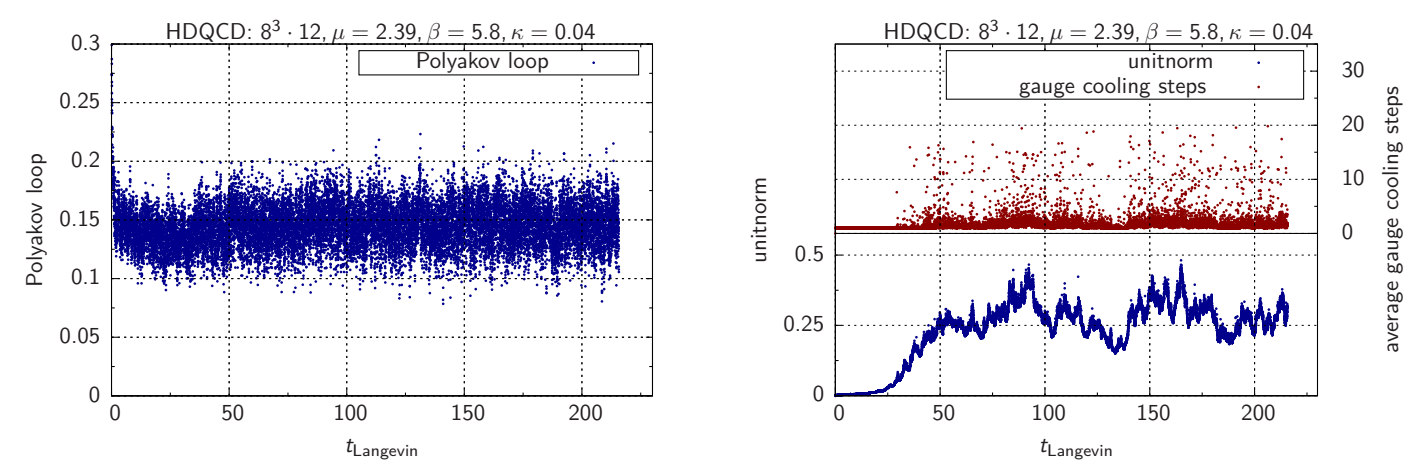

Figure 2: Left: The real part of the Polyakov loop $P$ as function of the Langevin time $t$. Right: The unitarity norm, i.e. the distance of the gauge links from the $\mathrm{SU}(3)$ manifold (below) and the average number of gauge cooling steps in a small Langevin time interval used between each Langevin update (above) as a function of Langevin time.

\section{Strategy and simulations details}

We perform complex Langevin simulations for fixed gauge coupling, volume and hopping parameter, more precisely we use the following setup:

- Volume: $8^{3} \times N_{\tau}$

- $N_{f}=2$

- $\beta=5.8$ 
- $\kappa=0.04$

- $N_{\tau}=2,3,4,5,6,7,8,9,10,12,14,15,16,18,20,24,28$

- $\mu=0.0-3.6$

The corresponding lattice spacing $a \sim 0.15 \mathrm{fm}$ has been determined using the gradient flow $[12,13]$. In the heavy dense theory the quark mass is given by

$$
m_{q} \equiv-\ln (2 \kappa)=2.53 \sim \mu_{c}
$$

which also corresponds to the critical chemical potential $\mu_{c}$, i.e. the chemical potential where the onset occurs at zero temperature. We vary the chemical potential for fixed temporal extent $N_{\tau}$, or equivalently fixed temperature $T$, to determine the transition to high densities by studying the fermion density,

$$
n=\frac{1}{N_{\tau} N_{s}^{3}} \frac{\partial \ln Z}{\partial \mu} .
$$

For the thermal deconfinement transition we simulate different temperatures for a given chemical potential $\mu$ and look at the behaviour of the Polyakov loop $P_{\vec{x}}$. A graphical illustration of this strategy is shown in figure 3. In total we have simulated 1024 different combinations of $N_{\tau}$ and $\mu$. These simulations have been extended to at least 100 units in Langevin time, so that we have roughly 2000 independent measurements taking into account thermalisation and auto-correlation.

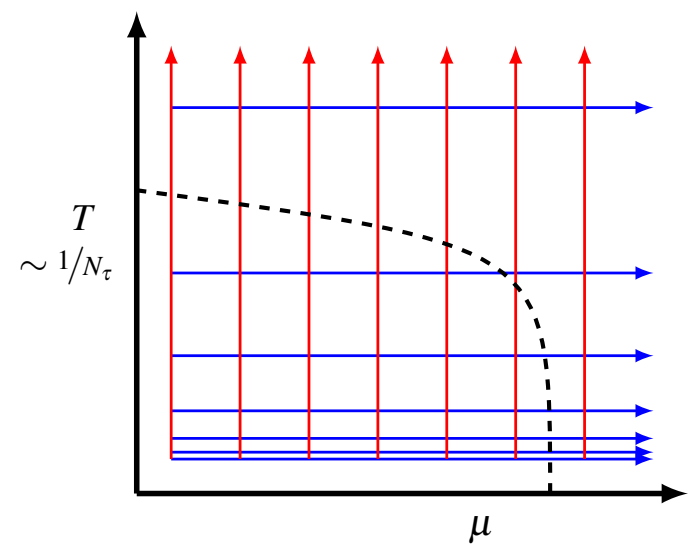

Figure 3: Strategy for determining the phase diagram of heavy dense QCD. The red and blue lines indicate scans for fixed $\mu$ or fixed temperature $T$, at a given $\beta$ and $\kappa$.

\section{Results}

Figure 4 shows the fermion density as a function of the chemical potential for 4 different temporal extents or temperatures. The transition to high densities can be seen around the predicted value for the critical chemical potential $\mu_{c}$. As expected the transition becomes sharper for lower temperatures. The saturation to $n_{\mathrm{sat}}=2 \cdot N_{c} \cdot N_{f}=12$ in figure 4 is a pure lattice artefact: As soon as every lattice site has been filled with 12 fermions, Pauli blocking prohibits more fermions to 


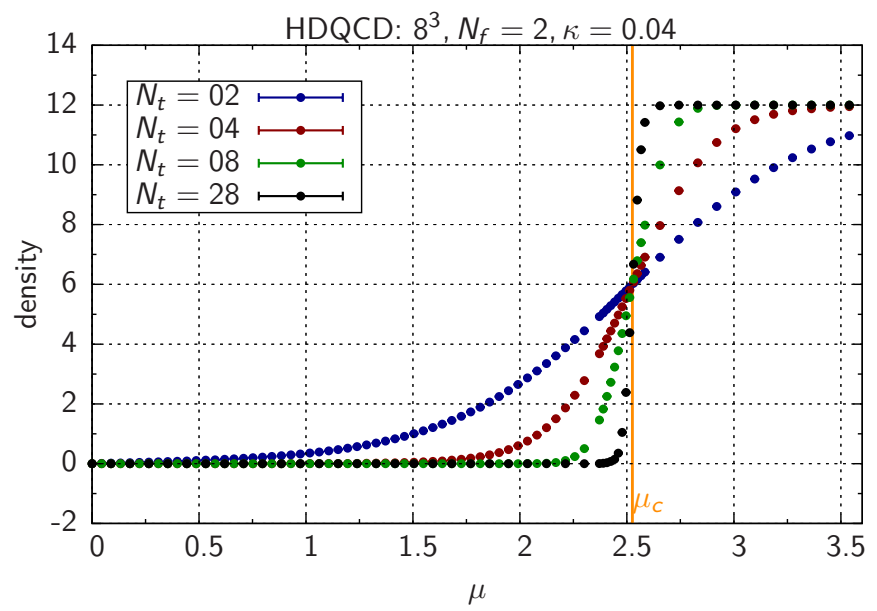

Figure 4: The fermion density as a function of the chemical potential $\mu$. The expected critical chemical potention $\mu_{c}=m_{q}=-\ln (2 \kappa)$ is shown as well.

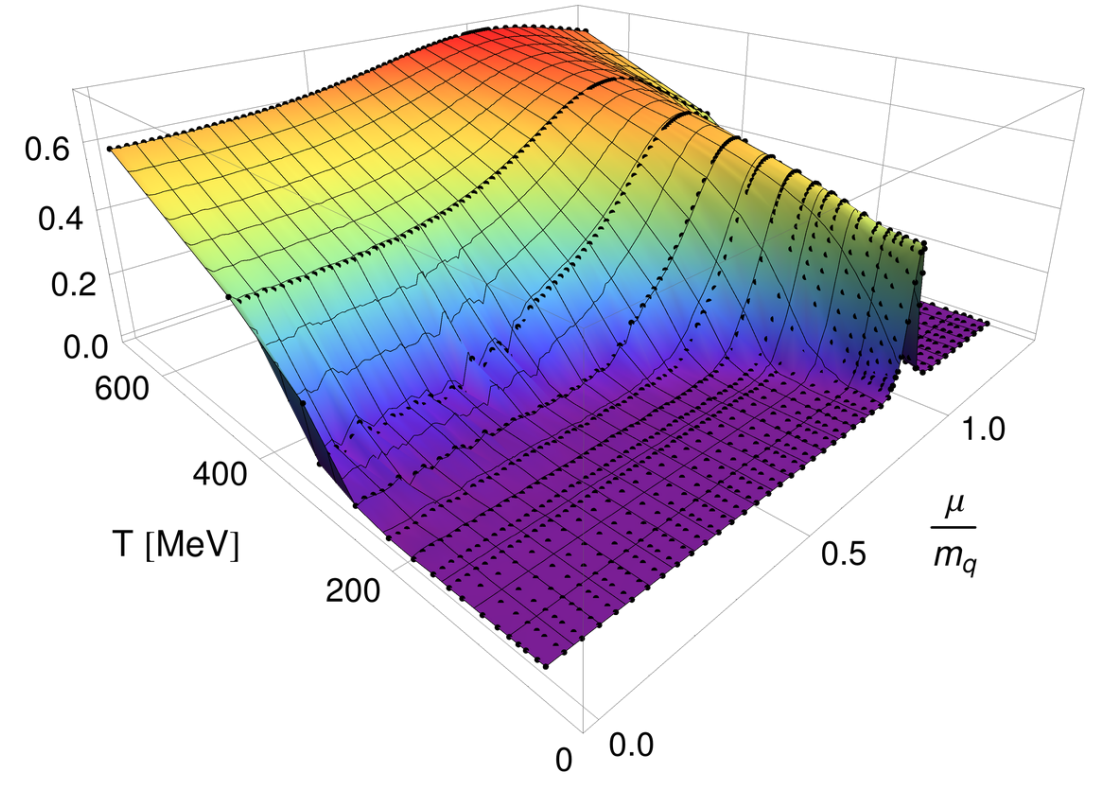

Figure 5: The real part of the Polyakov loop as function of the temperature and chemical potential for fixed $\beta$ and $\kappa$. The black points are the results of complex Langevin simulations for a given value of $\mu$ and $T$. The surface is the result of a cubic interpolation, in which the value of the Polyakov loop is encoded in the colour.

be added. Results for the Polyakov loop are shown in figure 5 as a function of $\mu$ and $T$. Each black point represents the results from one Langevin simulation. The coloured surface is the result of a cubic interpolation to estimate the intermediate behaviour, since the resolution in temperature is limited due to the discrete steps for the temporal extent. As the temperature is increased, the Polyakov loop shows the thermal deconfinement transition at fixed $\mu$. At fixed $T$, increasing $\mu$ leads to the transition to the high-density phase. The subsequent drop of the Polyakov loop, beyond $\mu / m_{q} \simeq 1$, is a lattice artefact due to saturation.

To identify the order of the transition we have started to study susceptibilities of these observ- 
ables. The result for the Polyakov loop susceptibility is shown in figure 6. The boundary of the

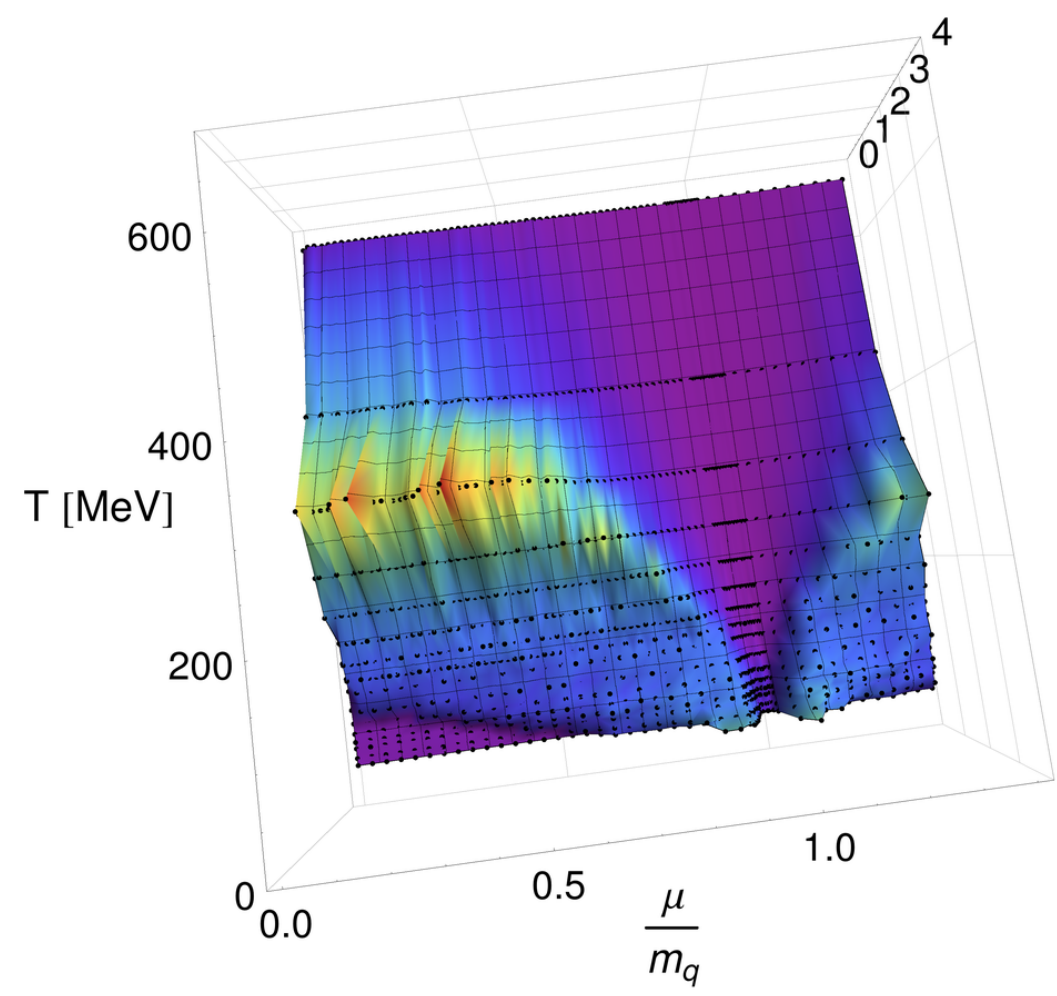

Figure 6: The Polyakov loop susceptibility as function of the temperature and chemical potential for fixed $\beta$. The black points are the results of complex Langevin simulations for a given value of $\mu$ and $T$. The surface is the result of a cubic interpolation, in which the value of the Polyakov loop susceptibility is encoded in the colour.

phase diagram is clearly visible. At $\mu / m_{q} \simeq 1$ the Polyakov loop has a maximum and the susceptibility vanishes. However, as mentioned above, this is a lattice artefact. On the other hand, the peak in the susceptibility at lower $\mu$-values is physical and indicates the transition to the deconfined phase. Intriguingly, at very low temperature and chemical potential there is an indication that the Polyakov loop and its susceptibility increase from zero while still in the confined phase. This requires further study. More simulations with different gauge couplings will allow us to study the intermediate region and improve the resolution for higher temperatures. By varying the volume and computing Binder cumulants [14] we will be able to determine the order of the phase transition as $\mu$ and $T$ are varied.

\section{Conclusions and Outlook}

Lattice simulations using the complex Langevin method deliver a reliable method to determine the phase diagram for QCD in the limit of heavy quarks. Future improvements include the variation of the simulation volume and the gauge coupling in order to determine the phase diagram more accurately as well as the order of the phase transition. The study of heavy dense QCD serves both as a guideline for future studies of "full" QCD [12] including dynamical fermions, as well as a proof of principle. 
Acknowledgments: We are grateful for the computing resources made available by HPC Wales and by STFC through DiRAC computing facilities. This work is supported by STFC, the Royal Society, the Wolfson Foundation and the Leverhulme Trust. FA is grateful for the support through the Brazilian government program "Science without Borders" under scholarship number Bex 9463/13-5.

\section{References}

[1] G. Aarts, PoS LATTICE 2012 (2012) 017.

[2] G. Parisi, Phys. Lett. B 131 (1983) 393.

[3] J. R. Klauder, Acta Phys. Austriaca Suppl. 25 (1983) 251.

[4] J. R. Klauder, Phys. Rev. A 29 (1984) 2036.

[5] G. Aarts and I. O. Stamatescu, JHEP 0809 (2008) 018.

[6] G. Aarts, F. A. James, E. Seiler and I. O. Stamatescu, Phys. Lett. B 687 (2010) 154.

[7] E. Seiler, D. Sexty and I. O. Stamatescu, Phys. Lett. B 723 (2013) 213.

[8] G. Aarts, L. Bongiovanni, E. Seiler, D. Sexty and I. O. Stamatescu, Eur. Phys. J. A 49 (2013) 89.

[9] L. Bongiovanni, G. Aarts, E. Seiler, D. Sexty and I. O. Stamatescu, PoS LATTICE 2013 (2013) 449.

[10] G. Aarts, E. Seiler and I. O. Stamatescu, Phys. Rev. D 81 (2010) 054508.

[11] G. Aarts, F. A. James, E. Seiler and I. O. Stamatescu, Eur. Phys. J. C 71 (2011) 1756.

[12] D. Sexty, Phys. Lett. B 729 (2014) 108.

[13] S. Borsanyi, S. Durr, Z. Fodor, C. Hoelbling, S. D. Katz, et al., JHEP 1209 (2012) 010.

[14] K. Binder, Z. Phys. B 43 (1981) 119. 